\title{
General Equilibrium and Endogenous Creation of Asset Markets.
}

\author{
MARTA FAIAS* \\ Departamento de Matemática, Faculdade de Ciências e Tecnologia, \\ Universidade Nova de Lisboa.
}

Running title: Endogenous Creation of Asset Markets.

Current address: Departamento de Matemática, Faculdade de Ciências e Tecnologia, Universidade Nova de Lisboa, Quinta da Torre, 2825-114 Monte da Caparica, Lisboa, PORTUGAL. Phone: 00-351-212948388 (Ext.10824). Fax: 00351-212948391. e-mail: mcm@fct.unl.pt

*I am specially grateful to my adviser Mário Páscoa for several helpful suggestions and discussions and I also thank Emma Moreno-García for valuable comments and conversations. Financial support from Fundação Amélia de Melo (Portugal) and from Portuguese Ministry of Science and Technology (Project SAPIENS 36525/99) is gratefully acknowledged. Faculdade de Ciências e Tecnologia da UNL, Monte da Caparica, 2825-114, Lisboa, Portugal. 
Abstract: This paper studies a class of general equilibrium economies in which asset markets arise as choice of financial intermediaries. The economy is modeled as a two stage game as in Bisin[8]. In the first stage intermediaries set up the financial structure according to the expectation that they have for the second stage outcome. In the second stage, consumers behave as price takers in the commodity market and in the previously created assets market. We consider that intermediaries form their expectations using continuous random selections from the second stage equilibrium correspondence (differently from Bisin [8] where an endogenous beliefs expectation was used). We establish the existence of equilibria in mixed strategies and moreover, we obtain an approximate equilibria in pure strategies by modeling explicitly the incomplete information that each intermediary has about others intermediaries fixed cost functions.

Key words: Endogenous asset creation, asset design game, strategic intermediaries, continuous random selections, purification of equilibria.

JEL Classification: G20, D51. 


\section{INTRODUCTION}

In the general equilibrium models with exogenously given financial markets, the properties of final allocations depend on the fixed structure of these assets. Our purpose is to provide a model where the asset structure arises endogenously as choice of economic agents. This issue was already studied in the literature and the papers by Allen and Gale [5], Duffie and Jackson [12], Duffie and Rahi [13], Hara [22] and Pesendorfer [29] constitute the first references to this topic. Nevertheless, the more general framework was developed in the paper by Bisin [8].

In Bisin [8], the economy is composed by two kind of agents, intermediaries that are imperfectly competitive and consumers that are price takers. The intermediaries design the payoff of the securities that they issue and choose the spread they charge on each security in order to maximize profits. A fixed cost and a proportional transaction cost are required to intermediate each security. Equilibrium prices are fixed by a Walrasian auctioneer to clear the spot and security markets and prices and demands are rationally anticipated by intermediaries. Then, the intermediaries are able to evaluate their profit for each financial structure, therefore, they are able to choose the financial structure that gives them an optimal profit. Using this setup Bisin obtains the existence of equilibrium in mixed strategies.

The framework of our work follows the setup of Bisin. However, we take up a different approach to solve the model. Besides, we obtain, not just the existence of equilibrium in mixed strategies, but also the existence of an approximate equilibrium in pure strategies. We remark that our tool can be applied also in other approaches, for instance, exchange economies where assets are designed by consumers. The models of Braido[9] and Faias[PhD diss] are examples of this class of economies.

In Bisin [8] and in this work the behavior of the intermediaries is modelled by a game. In this game the intermediaries maximize their expected payoff functions anticipating the Walrasian prices. Our model diverges from the model of Bisin in the way how the intermediaries form the expectations. More precisely, Bisin uses admissible beliefs and the Nash equilibrium existence results for games with upper-hemicontinuous profit correspondences due to Simon-Zame [32]. We use the random selection technique introduced by Allen [3] and explored by Mas- 
Colell and Nachbar [27], Allen [4] and Stahn [33] in models of imperfect competition in complete markets. We show the existence of a continuous random selection in the Walrasian equilibrium correspondence which implies the continuity of each player's payoff function. From this continuity we obtain the equilibrium in mixed strategies for the asset creation game. However, another important consequence of this continuity is that we can go one step further and obtain a pure strategy solution by explicitly modelling intermediaries' incomplete information with respect to competitors' fixed cost functions, within a family of linear functions of the mean and variance of the asset return. We apply the results of Milgrom and Weber [28], to get the existence of equilibrium in distributional strategies. Finally, exploring the Liapunov convexification theorem, we obtain the generic existence of an approximate equilibrium in pure strategies (which associates to each type a pure strategy).

The paper is organized as follows. The model is introduced in section 2. In section 3 we define the equilibrium concept and present the result of existence of equilibrium. In section 4 we introduce incomplete information and establish the existence of distributional strategy equilibrium and the generic existence of an approximate equilibrium in pure strategies.

\section{THE ECONOMY}

The model has two stages with two periods in the second stage, $t=0,1$. Uncertainty on the states of nature over the period 1 is denoted by $s \in S=1,2, \ldots, S$. There are $l=1, \ldots, L$ commodities in each periods and state. Let $n=(S+1) L$ be the number of states and time contingent goods of the economy.

\section{Consumers.}

Each consumer $i$, for $i=1, \ldots, I$ is characterized by an utility function $u^{i}$ : $\mathbb{R}_{+}^{n} \longrightarrow \mathbb{R}$ and by an initial endowment vector $w^{i}=\left(w_{0}^{i}, w_{1}^{i}, \ldots, w_{S}^{i}\right) \in \mathbb{R}_{+}^{n}$.

We state the following assumptions for the utility functions:

(A.1) $u_{i}$ is twice differentiable, $D u_{i}>>0$, and $D^{2} u_{i}$ is negative definite on $\mathbb{R}_{++}^{n}$.

(A.2) The closure of each indifference curve of $u_{i}$ does not intersect the boundary of $\mathbb{R}_{++}^{n}$. 
(A.3) $u_{i}$ is monotonic in commodity $1(s)$ for each $s \in S$ : Let $\tilde{x}$ be any consumption plan that is nonnegative in every coordinate, and strictly positive at $1(s)$ for some $s \in S$. Then for all $x \in \mathbb{R}_{++}^{n}, u_{i}(x+\tilde{x})>u_{i}(x)$.

\section{Intermediaries.}

We consider that in this economy there are $h=1, \ldots, H$ intermediaries who design assets and provide financial transactions. These designers are alive only at period 0 , are endowed with the good 1 and have utility just on this good. Each security in the economy is characterized by a payoff vector $a=\left(a_{1}, \ldots, a_{S}\right)$, where $a_{s}$ for each state of nature $s$ is a numeraire return denominated in units of good 1 . We assume that each intermediary can issue at most $J^{h}$ assets, which is an exogenous given number. Let $J=\sum_{h=1}^{H} J^{h}$ and let $a_{j}, j=1, \ldots, J$ denote the tradable assets in the economy. To create an asset the intermediary have to pay fixed issuing costs, and transaction costs, and obtain as return a bid-ask spread. For any asset $a \in \mathbb{R}^{S}$, the functions $\varepsilon(a)$ and $c(a)$ denote respectively variable costs and fixed costs associated to the creation of the asset $a$, and both are denominated in units of commodity 1 . We state the following assumptions for the cost functions:

(B.1) $\varepsilon(a), c(a) \geq 0 ;=0$ iff $a=0$.

(B.2) $\varepsilon(a), c(a)$ are, respectively, homogenous of degree 1 and 0 in $a$.

(B.3) $\varepsilon(a), c(a)$ are twice differentiable.

Assumption (B.2) means that issuing two identical assets with payoff $a$ has the same total cost of issuing one asset with payoff $2 a$. We assume that the return of each asset in each state of nature is nonnegative, that is, $a_{j}(s) \geq 0$ for $j=1, \ldots, J$ and $s=1, \ldots, S$. The assumption (B.2) implies that without loss of generality, the set of securities payoff can be normalized to:

$$
\mathcal{A}=\left\{a \in \mathbb{R}_{+}^{S}: \max _{s \in S} a(s)=1 \text { or } a(s)=0, \text { for all } s \in S\right\}
$$

The asset return structure is then represented by a matrix $A=\left\{a_{j}(s)\right\}_{s \in S}^{j \in J}$ which is a $S \times J$ matrix with $A \in \mathcal{A}^{J}=\mathcal{A} \times \cdots \times \mathcal{A}$. Given an asset structure $A$ we denote by $\hat{J}$ the maximal number of independent columns of this matrix, that 
is, the minimal number of assets that span the space spanned by this financial structure.

The bid-ask spread for each asset $a$ is described by a non linear function that we assume with the form $G(\gamma)(z)=\gamma z^{2}$. The bid ask spread function is the same for all assets apart from the parameter $\gamma$ which is also a choice of the intermediary that design the asset. We assume that the spread parameter $\gamma$ is chosen in a certain set of admissible spread parameters that, as in Bisin, we suppose compact. Let the set of admissible spread parameters be $\Gamma=[\underline{\gamma}, \bar{\gamma}]$, in the strictly positive axis. Since $\gamma>0$, by taking this spread function, we are assuming that the bid-ask spread on each asset is an increasing function of the quantity of the asset that is transacted. We also remark that since $G(\gamma)(z)>0$ if $z \neq 0$, then when a consumer buys an asset he pays more than its market price and when a consumer sells an asset he receives less than its market price. Spreads are also denominated in units of good 1.

These costs and this spread describe the technology that is available to create the asset markets structure in this economy.

We consider that the intermediary just have endowment and gets utility from the good 1 of the first period. The purpose of the intermediaries will be to maximize profits, that is, to maximize the difference between the spread and the costs of the asset. We denote by $z=\left(z_{1}, \ldots, z_{J}\right)$ a portfolio of assets in this economy and by $z_{+}=\left(z_{+1}, \ldots, z_{+J}\right)$ the corresponding portfolio of asset purchases. The payoff of the intermediary $h$ associated with a portfolio $z$ is:

$$
\pi^{h}=\sum_{j=1}^{J^{h}}\left[G\left(\gamma_{j}\right)\left(z_{j}\right)-\varepsilon\left(a_{j}\right)\left(z_{+j}\right)-c\left(a_{j}\right)\right] .
$$

\subsection{The Economy with an Asset Market Structure Fixed.}

Suppose that an economy $\left\{\left(u^{i}, w^{i}\right) ; i \in I\right\}$, a vector of spread parameters $\gamma$ and an asset return structure $A$ are fixed. Then, given $\left(p_{0}, p_{1}, q\right) \in \mathbb{R}_{+}^{n}+\mathbb{R}^{J}$, the utility maximization problem of the consumers is:

$$
\begin{array}{cl}
\max _{x \in \mathbf{R}_{+}^{n}, z \in \mathbf{R}^{J}} & u^{i}(x) \\
\text { s.a. } & p_{0}\left(x_{0}-w_{0}^{i}\right)+p_{01} G(\gamma)(z)+q z=0 \\
& p_{s}\left(x_{s}-w_{s}^{i}\right)=p_{1 s} a(s) z \quad \text { for all } \quad x \in \mathbb{R}_{+}^{n}, z \in \mathbb{R}^{J}
\end{array}
$$


where $G(\gamma)(z)=\sum_{j=1}^{J} G\left(\gamma_{j}\right)\left(z_{j}\right)$.

Definition 1. A Walrasian equilibrium for a given vector of spread parameters $\gamma$ and for a given asset return structure $A$ is a collection $\left(\bar{p}_{0}, \bar{p}_{1}, \bar{q},\left(\bar{x}^{i}, \bar{z}^{i}\right)_{i \in I},\left(\bar{x}^{h}\right)_{h \in H}\right) \in$ $\mathbb{R}_{+}^{n} \times \mathbb{R}^{J} \times\left(\mathbb{R}_{+}^{n} \times \mathbb{R}^{J}\right)^{I} \times \mathbb{R}^{H}$ such that:

(a) For every consumer $i,\left(\bar{x}^{i}, \bar{z}^{i}\right)$ solves the consumer problem.

(b) For every intermediary $h, \bar{x}^{h}=\sum_{j=1}^{J^{h}} \sum_{i=1}^{I}\left[G\left(\gamma_{j}\right)\left(\bar{z}_{j}^{i}\right)-\varepsilon\left(a_{j}\right) \bar{z}_{j}^{i}-c\left(a_{j}\right)\right]+w^{h}$.

(c) Markets clear,

$$
\begin{aligned}
\sum_{i=1}^{I}\left(\bar{x}_{10}^{i}-w_{10}^{i}\right)+\sum_{h=1}^{H}\left(\bar{x}^{h}-w^{h}\right)+\sum_{j=1}^{J}\left[\varepsilon\left(a_{j}\right)\left(\sum_{i=1}^{I} \bar{z}_{j}^{i}\right)+c\left(a_{j}\right)\right] & =0 \\
\text { For all } l \neq 1, \quad \sum_{i=1}^{I}\left(\bar{x}_{l 0}^{i}-w_{l 0}^{i}\right) & =0 \\
\text { For all } s, \quad \sum_{i=1}^{I}\left(\bar{x}_{s}^{i}-w_{s}^{i}\right) & =0 \\
\sum_{i=1}^{I} \bar{z}^{i} & =0 .
\end{aligned}
$$

Lemma 1. If assumptions A.1. - A.3. and B.1. - B.3. are satisfied, there exist a Walrasian equilibrium for a given vector of spread parameters $\gamma$ and for a given asset return structure $A$.

The main theorem of the next section requires that the aggregate demand function be continuously differentiable. The $S+1$ occurrences of the walras law allow us to drop $S+1$ markets, and if one of that markets is precisely the market of the commodity 1 in the first period then the aggregate excess demand function in the remaining markets can be written has:

$$
f\left(w, \gamma, A, p_{0}, p_{1}, q\right)=\left(x\left(w, \gamma, A, p_{0}, p_{1}, q\right), z\left(w, \gamma, A, p_{0}, p_{1}, q\right)\right)-\left(\sum_{i \in I} w^{i}, 0\right) .
$$

Lemma 2. If assumptions $(A .1)-(A .3)$ and $(B .1)-(B .3)$ are satisfied, individual demand is a $C^{1}$ function.

By homogeneity in prices we can take as the set of prices the simplex $\Delta^{(L-1) S} \times$ $\Delta^{L+J-1}$ with $\Delta^{L-1}=\left\{p(s) \in \mathbb{R}_{+}^{L}: \sum_{l \in \mathrm{E}} p_{l}(s)=1\right\}$ and $\Delta^{L+J-1}=\left\{\left(p_{0}, q\right) \in \mathbb{R}_{+}^{L+J}\right.$ : 
$\left.\sum_{l \in L, j \in J} p_{0 l}+q_{j}=1\right\}$. Therefore the excess demand function $f\left(w, \gamma, A, p_{0}, p_{1}, q\right)$ is a $C^{1}$ function in the interior of the price set $\Delta^{(L-1) S} \times \Delta^{L+J-1}$.

\subsection{Asset Creation.}

At the first stage of the model each intermediary strategically chooses the payoff structure and the spread parameter of some pre-fixed number of assets. The intermediaries have rational expectations which mean that they correctly anticipate the equilibrium price set corresponding to each vector of spread parameters and to each payoff matrix, therefore the intermediaries know the corresponding set of payoffs. The goal of the intermediaries is to choose the vector of spread parameters and the payoff matrix that maximizes their individual payoff. However, if we consider that intermediaries evaluate their payoffs through this correspondence, we face a difficulty because this correspondence is not continuous. To overcome this problem, we apply the random selections technique which was developed by Allen [3], Mas-Collel and Nachbar [27] and H. Stahn [33].

The equilibrium correspondence which maps a vector of spread parameters $\gamma$ and a matrix $A$ into the related set of Walrasian prices that arises in the second stage of the economy, is defined using the aggregate demand function as follows:

$$
E: \Gamma^{J} \times \mathcal{A}^{J} \longrightarrow 2^{\Delta^{(L-1) S} \times \Delta^{L+J-1}}
$$

with

$$
E(\gamma, A)=\left\{\left(p_{0}, p_{1}, q\right) \in \Delta^{(L-1) S} \times \Delta^{L+J-1}: f\left(w, \gamma, A, p_{0}, p_{1}, q\right)=0\right\}
$$

This correspondence is not continuous essentially due to jumps in the cardinality of the equilibrium set. If we adopt the random selections technique, this problem disappears. In fact, if one has in mind that the selection represents the inherent uncertainty about the market clearing price levels, we can assume that each consumer assigns a zero probability to a price level on a fold (unstable structure) because any small mistake in the price observation can lead to drastic errors in the evaluation of the indirect utility, i.e., in evaluating the payoffs.

Following the approach of Mas-Collel and Nachbar [27] the main tool to obtain a continuous random selection in the equilibrium is the Michael selection theorem . The Michael selection theorem states that a correspondence from a 
paracompact space to a complete, metrizable, locally convex, linear space, which is lower hemicontinuous and takes (non-empty) closed and convex values admits a continuous selection [see C. Aliprantis and K. Border [1]]. We will consider the subcorrespondence of quasi-regular equilibrium (this concept will be defined later) and prove that this subcorrespondence verifies the Michael selection theorem, then the subcorrespondence admits a continuous random selection which, actually, is a continuous random selection of the equilibrium correspondence $E$. We start by proving that for a residual economy the equilibrium correspondence $E$ maps each vector of spread parameters and payoff matrix into a countable set. This result is essential to prove that the equilibrium subcorrespondence is non-empty.

Given an asset structure $A$, we split the payoff matrix, $A=\left[\hat{A} \mid \hat{A}^{c}\right]$, where $\hat{A}$ is the submatrix of $A$ with the maximal number of independent columns and $\hat{A}^{c}$ is the submatrix with the remaining columns. We are considering that the columns of the matrix are ordered in such a way that the first columns are independent. The columns of $\hat{A}^{c}$ are in the space generated by the columns of $\hat{A}$, then, there exist a matrix $B$ such that $\hat{A}^{c}=\hat{A} B$. Thus, given a portfolio vector $z=\left(\hat{z}^{1}, \hat{z}^{2}\right)=\left(z_{1}, \ldots, z_{\hat{J}}, z_{\hat{J}+1}, \ldots, z_{J}\right)$, we define the adjusted portfolio $\hat{J}$-dimensional as $\hat{z}=\hat{z}^{1}+B \hat{z}^{2}$. It follows that, we can obtain the return vector $A z$ as the product between the payoff matrix $\hat{A}$ and the adjusted portfolio $\hat{z}$, that is, $A z=\left[\hat{A} \mid \hat{A}^{c}\right]\left[\hat{z}^{1} \hat{z}^{2}\right]^{\prime}=\hat{A}\left[\hat{z}^{1}+B \hat{z}^{2}\right]=\hat{A} \hat{z}$.

Let $\hat{f}\left(w, \gamma, A, p_{0}, p_{1}, q\right)$ be the aggregate demand function, where the aggregate portfolio is replaced by the aggregate adjusted portfolio. Let $\hat{E}(\gamma, A)=$ $\left\{\left(p_{0}, p_{1}, q\right) \in \Delta^{(L-1) S} \times \Delta^{L+J-1}: \hat{f}\left(w, \gamma, A, p_{0}, p_{1}, q\right)=0\right\}$, then $E(\gamma, A) \subseteq$ $\hat{E}(\gamma, A)$. We first show that the number of equilibra in $\hat{E}(\gamma, A)$ is countable, hence, we conclude that the number of equilibria in $E(\gamma, A)$ is at most countable.

An equilibrium price $\left(p_{0}, p_{1}, q\right)$ is a regular (resp. critical) equilibrium if $\hat{f}\left(w, A, \gamma, p_{0}, p_{1}, q\right)=0$ and $\operatorname{rank} D_{p_{0}, p_{1}, q} \hat{f}\left(w, A, \gamma, p_{0}, p_{1}, q\right)=(L-1)(S+1)+\hat{J}$ $($ resp. $<(L-1)(S+1))+\hat{J})$.

The next result states that the number of critical equilibria in $\hat{E}(\gamma, A)$ is finite for a residual set of utility functions and endowments of commodity $l=L$ for $\hat{J}$ states of one of the consumers. Thus, we have to perturb the utility function of one of the consumers [as in Mas-Colell and Nachbar [27]] but we have also to perturb the endowments of one consumer (in at least as many states as the 
number of assets) in one of the commodities.

Let us denote by $\mathcal{U}$ the space of utility functions of each consumer and endow the space with the topology of $C^{\infty}$ uniform convergence on compacta. It is then topologically complete and, therefore, residual subsets (i.e., sets containing the intersection of a countable collection of open dense sets) are dense.

For the next results the utility of the first consumer will be perturbed, thus, we fix all the utility functions $u_{2}, \ldots, u_{I}$ of consumers $i=2, \ldots, I$. Since we will perturb the endowment of the first consumer in the last good for $\hat{J}$ states of nature, we split the endowment vector of the consumers, $w=\left(w_{L \hat{J}}^{1}, w_{-L \hat{J}}^{1}\right)$ where $w_{L \hat{J}}^{1}=\left(w_{L}^{1}(s)\right)_{s=1}^{\hat{J}} \in \mathbb{R}_{+}^{\hat{J}}$ is the endowment in the commodity $L$ of the first consumer but just for the first $\hat{J}$ states of nature of the second period, and $w_{-L \hat{J}}^{1} \in \mathbb{R}_{+}^{L(1+S-\hat{J})+(L-1) \hat{J}+n(I-1)}$ enclose the remaining endowment vector of the consumers, namely, the remaining endowment vector of the first consumer (the endowment of the first period and the endowment of the last $S-\hat{J}$ states of nature of the second period in all commodities and the endowments of the states $s=1, \ldots, \hat{J}$ in the goods 1 to $L-1$ ), and the total endowment vectors of consumers $i=2, \ldots, I$.

The method of prove that we can find in [in Faias, Moreno-Garcia and Páscoa [16], Proposition 4.1.] and [Mas-Collel-Nachbar, [27], Proposition 1] when adapted to our context implies the following result.

Proposition 1. There is a set $\mathcal{U}^{*} \times \mathcal{W}_{L \hat{J}}^{*}$ residual in $\mathcal{U} \times \mathbb{R}_{+}^{\hat{J}}$ such that for an economy $\left\{\left(u^{i}\right)_{i \in I},\left(w_{L \hat{J}}^{1}, w_{-L \hat{J}}^{1}\right),\left(u^{h}, w^{h}\right)_{h \in H}, \gamma, A\right\}$ with $u_{1} \in \mathcal{U}^{*}, w_{L \hat{J}}^{1} \in \mathcal{W}_{L \hat{J}}^{*}$ and with an exogenous vector of spread parameters $\gamma \in \Gamma^{J}$ and an exogenous asset market given by the payoff matrix $A \in \mathcal{A}^{J}$ the number of critical equilibria in $\hat{E}(\gamma, A)$ is at most $n I+S J+J$.

The Inverse Function Theorem guarantees that regular equilibria are locally isolated. Therefore the number of equilibria in $\hat{E}(\gamma, A)$ is at most countable. Since, $E(\gamma, A) \subseteq \hat{E}(\gamma, A)$, we can state the required result.

Corollary 1. There is a set $\mathcal{U}^{*} \times \mathcal{W}_{L \hat{J}}^{*}$ residual in $\mathcal{U} \times \mathbb{R}_{+}^{\hat{J}}$ such that for an economy $\left\{\left(u_{i}\right)_{i=1}^{I},\left(w_{L \hat{J}}^{1}, w_{-L \hat{J}}^{1}\right), A, \gamma\right\}$ with $u_{1} \in \mathcal{U}^{*}, w_{L \hat{J}}^{1} \in \mathcal{W}_{L J}^{*}$ and with an exogenous vector of spread parameters $\gamma \in \Gamma$ and an exogenous asset market given by the payoff matrix $A \in \mathcal{A}$, the number of equilibria for the economy is at most countable. 
Let $\mathcal{P}\left(\Delta^{(L-1) S} \times \Delta^{L+J-1}\right)$ be the set of probabilities measures on $\Delta^{(L-1) S} \times$ $\Delta^{L+J-1}$.

Definition 2. A continuous random selection of $E$ is a function $\rho: \Gamma^{J} \times \mathcal{A}^{J} \longrightarrow$ $\mathcal{P}\left(\Delta^{(L-1) S} \times \Delta^{L+J-1}\right)$, such that: $(i)$ for all $(\gamma, A) \in \Gamma^{J} \times \mathcal{A}^{J}, \rho(\gamma, A)(E(\gamma, A))=1$ and (ii) $\rho$ is continuous, that is, if $\left(\gamma_{n}, A_{n}\right) \longrightarrow(\gamma, A)$ then $\rho\left(\gamma_{n}, A_{n}\right) \longrightarrow \rho(\gamma, A)$ for the topology of weak convergence.

Definition 3. An equilibrium point $\left(p_{0}, p_{1}, q\right)$ of $E(\gamma, A)$ is a quasi-regular equilibrium if for any $\varepsilon>0$ there exists some closed ball $B_{\varepsilon} \subset \Delta^{(L-1) S} \times \Delta^{L+J-1}$, centered at $\left(p_{0}, p_{1}, q\right)$ and of radius less then $\varepsilon$, such that the degree of $f(w, \gamma, A, ., .,$. restricted to $\partial B_{\varepsilon}$ is well defined (i.e., $0 \notin f\left(w, \gamma, A, \partial B_{\varepsilon}\right)$ and non-zero. [By degree of $f(w, \gamma, A, ., .$.$) on \partial B_{\varepsilon}$ we mean the degree of the map

$$
\left(p_{0}, p_{1}, q\right) \longrightarrow\left[1 /\left\|f\left(w, \gamma, A, p_{0}, p_{1}, q\right)\right\|\right] f\left(w, \gamma, A, p_{0}, p_{1}, q\right)
$$

viewed as a map of the sphere into itself.]

Definition 4. We denote by $E^{\prime}$ the subcorrespondence of the equilibrium correspondence $E$ which maps $\Gamma^{J} \times \mathcal{A}^{J}$ into the quasi-regular equilibria, that is, $E^{\prime}(\gamma, A)=\left\{\left(p_{0}, p_{1}, q\right) \in \Delta^{(L-1) S} \times \Delta^{L+J-1}: f\left(w, \gamma, A, p_{0}, p_{1}, q\right)=0\right.$ and $\left(p_{0}, p_{1}, q\right)$ is a quasi-regular equilibrium $\}$.

Proposition 2. If for every $(\gamma, A) \in \Gamma^{J} \times \mathcal{A}^{J}, E(\gamma, A)$ is at most countable than there exists a continuous random selection in the equilibrium correspondence $E$.

Proof: To prove this proposition, actually we will prove the existence of a continuous random selection for the subcorrespondence $E^{\prime}$, but this result trivially implies the existence of a continuous random selection to the correspondence $E$. The prove follows with three claims.

First claim: $E^{\prime}(A)$ is non empty.

By Cass trick [see Geanakoplos [18] and Magill and Shafer [25]] we can consider, without loss of generality, that the problem of one of the consumers is a problem with complete markets. Precisely, regarding commodities demand functions, we can consider that the budget constraint for such a consumer is defined by $p_{0}\left(x_{0}-w_{0}\right)+\sum_{s} \tilde{p}_{s}\left(x_{s}-w_{s}\right)=0$, being $\tilde{p}_{s}=\beta_{s} p_{s}$ and $\pi_{b}=\sum_{s} \beta_{s} r^{b}(s)$, for some $\beta \gg 0$. In this way, we have that if $\left(p_{0 n}, p_{1 n}, q_{n}\right)$ is a sequence of prices belonging 
to $E(\gamma, A)$ and converging to $\left(p_{0}, p_{1}, q\right)$, then the price system $\left(p_{0}, p_{1}, q\right)$ has every component non null. We conclude that $E(\gamma, A)$ is a compact set. Since we have also proved that $E(\gamma, A)$ is at most countable, it can be covered by finite many disjoint open balls $B_{k}, k=1, \ldots, K$, such that their closure are non-intersecting, [see Mas-Collel, Nachbar, [27], lemma 6].

On the other hand, we can take a set (diffeomorfic to a ball) close enough to the boundary of $\Delta^{(L-1) S} \times \Delta^{L+J-1}$, where $f(w, \gamma, A, ., .,$.$) does not change its$ orientation and therefore $f(w, \gamma, A, ., .$.$) has degree different from zero on such a$ set. Now we can take a sequence of balls $B^{n}$ with radius strictly decreasing and converging to zero and such that $f(w, \gamma, A, . . .,$.$) is well defined on \partial B^{n}$ and has non-zero degree. Thus $\cap B^{n}$ consists of a single point which by construction is a quasi-regular equilibrium, [see Mas-Collel Nachbar, [27], lemma 7].

Second claim: The correspondence $E^{\prime}: \Gamma^{J} \times \mathcal{A}^{J} \longrightarrow 2^{\Delta^{(L-1) S} \times \Delta^{L+J-1}}$ is lower hemicontinuous.

Fix some arbitrary $(\gamma, A) \in \Gamma^{J} \times \mathcal{A}^{J}$ and take a sequence $\gamma_{n} \times A_{n}$. If $\left(p_{0}, p_{1}, q\right)$ is a quasi-regular zero of $E(\gamma, A)$ then for any $\varepsilon>0$ no matter small, there is a ball $B_{\varepsilon}$ centered at $\left(p_{0}, p_{1}, q\right)$ and of radius then $\varepsilon$ such that $f(w, \gamma, A, ., .,$.$) restricted$ to $\partial B_{\varepsilon}$ is of non-zero degree. By continuity, for $n$ large enough, $f\left(w, \gamma_{n}, A_{n}, ., .,.\right)$ is homotopic to $f(w, \gamma, A, . . .,$.$) on \partial B_{\varepsilon}$, hence $f\left(w, \gamma_{n}, A_{n}, . . .,.\right)$ has non zero degree on $\partial B_{\varepsilon}$. By the boundary theorem [see Hirsch [24], p.136] there exist a $\left(p_{0 n}, p_{1 n}, q_{n}\right)$ such that $f\left(w, \gamma_{n}, A_{n}, p_{0 n}, p_{1 n}, q_{n}\right)=0$ and then $\left(p_{0 n}, p_{1 n}, q_{n}\right)$ is a quasi-regular zero of $f\left(w, \gamma_{n}, A_{n}, . . .,.\right)$. Of course, $\left\|\left(p_{0 n}, p_{1 n}, q_{n}\right)-\left(p_{0}, p_{1}, q\right)\right\|<\varepsilon$. Since $\varepsilon$ is arbitrary we are done.

Let us now consider the correspondence $E^{\prime \prime}: \Gamma^{J} \times \mathcal{A}^{J} \longrightarrow 2^{\mathcal{P}\left(\Delta^{(L-1) S} \times \Delta^{L+J-1}\right)}$ which applies each payoff matrix and spread parameter into a set of probabilities measures, $E^{\prime \prime}(\gamma, A) \subset \mathcal{P}\left(\Delta^{(L-1) S} \times \Delta^{L+J-1}\right)$ with each measure $\rho \in E^{\prime \prime}(\gamma, A)$ verifying that $\rho\left(E^{\prime}(\gamma, A)\right)=1$.

Third claim: The correspondence $E^{\prime \prime}$ is lower hemicontinuous.

Let $\left(\gamma_{n}, A_{n}\right) \longrightarrow(\gamma, A)$ and let $\rho \in E^{\prime \prime}(\gamma, A)$. Fix an arbitrary $\varepsilon>0$. Choose a finite set $\left\{\left(p_{01}, p_{11}, q_{1}\right), \ldots,\left(p_{0 m}, p_{1 m}, q_{m}\right)\right\} \subset E^{\prime \prime}(\gamma, A)$ with

$$
\rho\left(\left(p_{01}, p_{11}, q_{1}\right), \ldots,\left(p_{0 m}, p_{1 m}, q_{m}\right)\right)>1-\varepsilon
$$

By the lower hemicontinuity of $E^{\prime}$, for each $1 \leq h \leq m$, we can take a sequence 
$\left(p_{0 h n}, p_{1 h n}, q_{h n}\right)$ converging to $\left(p_{0 h}, p_{1 h}, q_{h}\right)$, with $\left(p_{0 h n}, p_{1 h n}, q_{h n}\right) \in E^{\prime}\left(\gamma_{n}, A_{n}\right)$. Finally, for each $n$, take for $\rho_{n}$ a probability measure supported by $\left\{\left(p_{01 n}, p_{11 n}, q_{1 n}\right), \ldots,\left(p_{0 m n}, p_{1 m n}, q_{m n}\right)\right\}$ and such that

$$
\rho_{n}\left(\left(p_{0 h n}, p_{1 h n}, q_{h n}\right)\right) \geq \rho\left(\left(p_{0 h}, p_{1 h}, q_{h}\right)\right) \text { for every } 1 \leq h \leq m \text {. }
$$

Since $\varepsilon$ is arbitrarily small, we deduce the lower hemicontinuity of the correspondence $E^{\prime \prime}$.

The three claims proved above allow us to apply the Michael selection theorem and guarantee that there is a continuous selection in the correspondence $E^{\prime \prime}$, which is the desired continuous random selection for the equilibrium correspondence $E^{\prime}$ and finally it is also the continuous random selection for the correspondence $E$.

We are now in position to define the game played by the intermediaries in the first stage of the model. We denote by $\Gamma_{h} \times \mathcal{A}_{h}$ the strategy set for each intermediary $h=1, \ldots, H$, where $\Gamma_{h}=[\underline{\gamma}, \bar{\gamma}]^{J_{h}}$ and $\mathcal{A}_{h}=\mathcal{A}^{J_{h}}$. The continuous random selection of the equilibrium correspondence is the main device to define a continuous payoff function for each intermediary as the profit expected value. Given a profile of strategies for each intermediary $\left(A_{h}, \gamma_{h}, A_{-h}, \gamma_{-h}\right)$ where $\left(A_{h}, \gamma_{h}\right)$ denotes the strategy of intermediary $h$, and $\left(A_{-h}, \gamma_{-h}\right)$ denotes the strategy of the remaining intermediaries, the payoff function for intermediary $h$ will be:

$$
\begin{aligned}
& \pi^{h}: \Gamma_{h} \times \mathcal{A}_{h} \times \Gamma_{-h} \times \mathcal{A}_{-h} \longrightarrow \mathbb{R}_{+} \\
& \pi^{h}\left(\gamma_{h}, A_{h}, \gamma_{-h}, \mathcal{A}_{-h}\right)= \\
& \quad \int_{\Delta^{(L-1) S} \times \Delta^{L+J-1}}\left[\sum_{j=1}^{J^{h}} \sum_{i=1}^{I}\left(G\left(\gamma_{j}\right),\left(z_{j}^{i}\right)-\varepsilon\left(a_{j}\right) z_{j}^{i}-c\left(a_{j}\right)\right)+w^{h}\right] d \rho(\gamma, A)
\end{aligned}
$$

where $\rho(\gamma, A)$ is the continuous random selection that the intermediaries expect when they set the vector of spread parameters $\gamma$ and design the asset matrix $A$. The asset creation is then described by a simultaneous Nash game which is characterized by $\mathbb{G} \equiv\left\{\left(\Gamma_{h} \times \mathcal{A}_{h}, \pi^{h}\right) ; h \in H\right\}$

Definition 5. A pair $\left(\gamma^{*}, A^{*}\right) \in \Gamma^{J} \times \mathcal{A}^{J}$ is a pure strategy Nash Equilibrium for the asset design game $\mathbb{G} \equiv\left\{\left(\Gamma_{h} \times \mathcal{A}_{h}, \pi^{h}\right) ; h \in H\right\} \quad$ if $\quad \pi^{h}\left(\gamma_{h}^{*}, A_{h}^{*}, \gamma_{-h}^{*}, A_{-h}^{*}\right)=$ $=\max _{\left(\gamma_{h}, A_{h}\right) \in \gamma_{h} \times \mathcal{A}_{h}} \pi^{h}\left(\gamma_{h}, A_{h}, \gamma_{-h}^{*}, A_{-h}^{*}\right)$ for all $h=1, \ldots, H$. 
Proposition 3. There is a set $\mathcal{U}^{*} \times \mathcal{W}_{L \hat{J}}^{*}$ residual in $\mathcal{U} \times \mathbb{R}_{+}^{\hat{J}}$ such that for every economy $\left.\left\{\left(u^{i}\right)_{i \in I},\left(w_{L \hat{J}}^{1}, w_{-L \hat{J}}\right),\left(u^{h}, w^{h}\right)_{h \in H}\right)\right\}$ with $u_{1} \in \mathcal{U}^{*}$ and $w_{L \hat{J}}^{1} \in \mathcal{W}^{*}$ the associated asset design game $\mathbb{G} \equiv\left\{\left(\Gamma_{h} \times \mathcal{A}_{h}, \pi^{h}\right) ; h \in H\right\}$ has equilibrium in mixed strategies.

Proof: We have that conditions hold that are sufficient for the application of Glicksberg's existence theorem [see Fundenberg and Tirole [17]]. Namely, the strategy sets are compact and the payoff functions of each player are continuous.

\section{Equilibrium and the Main Result}

The equilibrium concept allows for the consistence between the two stages through which the economy evolves.

Definition 6. An equilibrium for an economy $\mathcal{E}=\left\{\left(\left(u^{i}, w^{i}\right)_{i \in I},\left(u^{h}, w^{h}\right)_{h \in H}\right)\right\}$ is a pair $(\sigma, \alpha)$ such that:

(i) $\sigma$ is an equilibrium in mixed strategies for the associated asset design game, $\mathbb{G} \equiv\left\{\left(\Gamma_{h} \times \mathcal{A}_{h}, \pi^{h}\right) ; h \in H\right\}$ with $\sigma=\sigma_{1} \times \cdots \times \sigma_{H}$ and with $\sigma_{h}$ being a probability over $\Gamma_{h} \times \mathcal{A}_{h}$;

(ii) $\alpha$ is a measure over the price set given by the Gelfand integral of the random selection with respect to the measure $\sigma$, that is, $\alpha=\rho((\Gamma, A)) d \sigma$.

Remark that for a function $g \in C\left(\Delta^{(L-1) S} \times \Delta^{L+J-1}\right)$ we have,

$$
\int_{\Delta^{(L-1) S} \times \Delta^{L+J-1}} g d(\rho((\Gamma, A)) d \sigma)=\int_{\Gamma \times \mathcal{A}} \int_{\Delta^{(L-1) S} \times \Delta^{L+J-1}} g d \rho((\Gamma, A)) d \sigma
$$

By words, we can say that the measure $\alpha$ is a price distribution which is the expected value of the continuous random selection with respect to the mixed strategies profile over vector of spread parameters and return matrices. This measure $\alpha$ exists because $\rho$ is continuous for the weak* topology in the range space and therefore, Gelfand integrable.

Theorem 1. There is a set $\mathcal{U}^{*} \times \mathcal{W}_{L \hat{J}}^{*}$ residual in $\mathcal{U} \times \mathbb{R}_{+}^{\hat{J}}$ such that for every economy $\left.\left\{\left(u^{i}\right)_{i \in I},\left(w_{L \hat{J}}^{1}, w_{-L \hat{J}}\right),\left(u^{h}, w^{h}\right)_{h \in H}\right)\right\}$ with $u_{1} \in \mathcal{U}^{*}$ and $w_{L \hat{J}}^{1} \in \mathcal{W}_{L \hat{J}}^{*}$, the equilibrium set is not empty.

Proof: The theorem follows from lemma 2 and proposition 10. 


\section{Asset Design with Incomplete Information: Purification of Equilibria}

In this section we show that for an appropriate economy with incomplete information we can guarantee the existence of equilibrium in distributional strategies and moreover, we guarantee the existence of approximate equilibrium in pure strategies. The existence of an approximate equilibrium in pure strategies follows from the Milgrom and Weber [28] theorem which asserts that for games in which the informational variables of the players have atomless distributions, each player's set of pure strategies is dense in his complete set of strategies, that is, in his set of distributional strategies.

The model in this context is similar to the previous model, namely, at the first stage of the model the intermediaries behave strategically choosing the matrix of asset market returns and the related vector of spread parameters. The intermediaries have rational expectations hence they correctly anticipate the equilibria price set corresponding to each financial structure and therefore they can evaluate the payoff associated to each financial structure. Then, we will consider as before a model with two stages. In the first stage we define a game in spreads and assets in a context of incomplete information. Using this incomplete information framework we obtain the purification of the equilibrium by exploring the Milgrom and Weber [28] results.

Each intermediary is characterized by an informational variable (or type) $t_{h}$, which defines the fixed cost associated to asset design and trade. Let us address the case of fixed cost functions given by, $c_{h}(a)=\alpha_{1 h}+\alpha_{2 h} M(\tilde{a})+\alpha_{3 h} V(\tilde{a})$ where $\tilde{a}=a / \max _{i}\left\{a_{i}\right\}$ and $M(\tilde{a})$ and $V(\tilde{a})$, denote the mean and the variance (taking up a uniform distribution on the states of nature) of the asset $\tilde{a}$, respectively. The use of $\tilde{a}$ instead of $a$ in the fixed cost definition assures that the assumption (B.2) on costs holds. The independent term $\alpha_{1 h}$ represents the cost of locating potential clients and the cost of setting up the business activity, the other term, $\alpha_{2 h} M(\tilde{a})+\alpha_{3 h} V(\tilde{a})$, represents the cost of designing and marketing the asset, as a function of the mean and variance of its returns. Thus, the fixed cost function of each intermediary, $c_{h}$, is described by the vector of parameters $\alpha_{h}=$ $\left(\alpha_{1 h}, \alpha_{2 h}, \alpha_{3 h}\right) \in \mathbb{R}_{+}^{3}$, that is $c_{h}=c_{h}\left(\alpha_{h}\right)$, and the vector $\alpha_{h}$ constitutes the informational variable (or type), $t_{h}=\alpha_{h}$. The informational structure in the set 
of types is defined by a probability measure $\eta$ on the Borel subsets of $\left(\mathbb{R}_{+}^{3}\right)^{H}$. Associated with the informational structure $\eta$ are the marginal distributions of each intermediary, $\eta_{h}$. We state the following assumptions for the informational structure:

(C.1) The informational variable $t_{h}$ belongs to a compact set $T_{h}=T \subset \mathbb{R}_{+}^{3}$ for every $h=1, \ldots, H$.

(C.2) The measure $\eta$ is absolutely continuous with respect to the measure $\hat{\eta}=$ $\eta_{1} \times \cdots \times \eta_{H}$.

In this context, with incomplete information, the second stage of the model proceeds exactly as the second stage of the previous model. The innovated securities and its related spreads are already fixed in the market, then, it is time to a Walrasian auctionner to determine the equilibrium prices and allocations for the economy. In fact, in the second stage of the model we can interpret the economy as an economy with exogenous given financial markets structure. The equilibrium prices would be such that each consumer and each intermediary maximizes utility. The problem is the same that we solved in section 2.1, therefore lemma 2 is suitable and it also holds that the excess demand function is a $C^{1}$ function.

The main focus of this approach with incomplete information comes out in the first stage of the model. The intermediaries design the asset market structure through a game with incomplete information. The strategy space of each playerintermediary, is the set $\mathcal{M}_{h}$ which denotes the set of probability measures over the Borel sets of $T \times \Gamma_{h} \times \mathcal{A}_{h}$. Let $\mathcal{M}=\mathcal{M}_{1} \times \ldots \times \mathcal{M}_{H}$.

Definition 7. A distributional strategy for player $h$ is a probability measure $\mu_{h}$, such that its marginal distribution on $T$ is the one specified by the information structure, $\eta_{h}$. Precisely, this restriction on the marginal distributions means that for all $S \subset T, \mu_{h}\left(S \times \Gamma_{h} \times \mathcal{A}_{h}\right)=\eta_{h}(S)$.

The payoff function of each intermediary stills being build up through the equilibrium correspondence. However we need to consider another argument in this correspondence. Let us denote by $\tilde{T}=T^{H}$ the set of intermediaries informational variable, which is the same as the set of intermediaries fixed cost functions. We denote by $\alpha=\left(\alpha_{1}, \ldots, \alpha_{H}\right) \subset \tilde{T}$ a vector of parameters that define the fixed 
cost functions of the intermediaries. The equilibrium correspondence in this approach is the correspondence $E_{I}: \tilde{T} \times \Gamma^{J} \times \mathcal{A}^{J} \longrightarrow \Delta^{(L-1) S} \times \Delta^{L+J-1}$ that applies a vector of fixed cost functions parameters $\alpha=\left(\alpha_{1}, \ldots, \alpha_{H}\right)$ and an asset market structure $(\gamma, A)$ into the corresponding walrasian prices, that is,

$$
E_{I}(\alpha, \gamma, A)=\left\{\left(p_{0}, p_{1}, q\right) \in \Delta^{(L-1) S} \times \Delta^{L+J-1}: f\left(\alpha, \gamma, A, p_{0}, p_{1}, q\right)=0\right\}
$$

Following the same steps of the proof of the proposition 9, we can also prove that the equilibrium correspondence $E_{I}$ contains a continuous random selection $\rho(\alpha, \gamma, A)$. Then, the expected payoff to each player-intermediary is constructed using the random selection in the same way of the previous section.

$$
\begin{gathered}
\pi^{h}: \mathcal{M}=\mathcal{M}_{1} \times \ldots \times \mathcal{M}_{H} \longrightarrow \mathbb{R}_{+} \\
\left(\mu_{1}, \ldots, \mu_{H}\right) \rightarrow \pi^{h}\left(\mu_{1}, \ldots, \mu_{H}\right)= \\
=\int_{\left(T \times \Gamma_{1} \times \mathcal{A}_{1}\right) \times \cdots \times\left(T \times \Gamma_{H} \times \mathcal{A}_{H}\right)} \int_{\Delta^{(L-1) S} \times \Delta^{L+J-1}}\left[\sum _ { j = 1 } ^ { J ^ { h } } \sum _ { i = 1 } ^ { I } \left(G\left(\gamma_{j}\right)\left(z_{j}^{i}\right)-\varepsilon\left(a_{j}\right) z_{j}^{i}-\right.\right. \\
\left.\left.c_{h}\left(\alpha_{h}\right)\left(a_{j}\right)\right)+w^{h}\right] d \rho(\alpha, A, \gamma) d \mu_{1} \cdots d \mu_{H}
\end{gathered}
$$

Therefore, given a numeraire asset market economy with incomplete information $\mathcal{E}_{I} \equiv\left\{\left(\left(u^{i}, w^{i}\right)_{i \in I},\left(u^{h},\left(T, \eta_{h}\right)\right)_{h \in H}\right)\right\}$, the asset market arise as a solution of the game $\mathbb{G}_{I} \equiv\left\{\left(\mathcal{M}_{h}, \pi^{h}\right), h \in H\right\}$ characterized by the strategy set $\mathcal{M}_{h}$ and by the payoff function $\pi^{h}$, for each intermediary $h=1, \ldots, H$.

Definition 8. A pure strategy for player $h$ is a measurable function $a_{h}: T \longrightarrow$ $\mathcal{A}_{h} \times \Gamma_{h}$. That is, a pure strategy is a distributional strategy $\mu_{h}$ whose conditional distributions $\mu_{h}(\backslash t)$ are Dirac measures for each type $t$.

Definition 9. An $\varepsilon$-equilibrium point of a game is a n-tuple $\left(\mu_{1}, \ldots, \mu_{n}\right)$ of player's strategies, such that for every player $i$ and every alternative strategy $\mu_{i}^{\prime}, \pi\left(\left(\mu_{1}, \ldots, \mu_{n}\right)\right)+$ $\varepsilon \geq \pi\left(\left(\mu_{1}, \ldots, \mu_{i}^{\prime}, \ldots, \mu_{n}\right)\right)$.

Proposition 4. There is a set $\mathcal{U}^{*} \times \mathcal{W}_{L \hat{J}}^{*}$ residual in $\mathcal{U} \times \mathbb{R}_{+}^{\hat{J}}$ such that for every economy $\mathcal{E}_{I} \equiv\left\{\left(\left(u^{i}, w^{i}\right)_{i \in I},\left(u^{h},\left(T, \eta_{h}\right)\right)_{h \in H}\right)\right\}$ with $u_{1} \in \mathcal{U}^{*}$ and $w_{L, \hat{J}}^{1} \in \mathcal{W}_{L \hat{J}}^{*}$ the associated game $\mathbb{G}_{I} \equiv\left\{\left(\mathcal{M}_{h}, \pi^{h}\right), h \in H\right\}$ has equilibrium in distributional strategies. If in addiction the measure $\eta_{h}$ is atomless for each $h=1, \ldots, H$, then, for every $\varepsilon>0$ the game has an $\varepsilon$-equilibrium in pure strategies.

Proof: The payoffs, $\pi^{h}$ of the game $\mathbb{G}_{I}$ are equicontinuous. For this, let $\left(\alpha^{n}, \gamma^{n}, A^{n}\right)$ be a sequence converging to $(\alpha, \gamma, A)$, then 


$$
\int_{\Delta^{(L-1) S} \times \Delta^{L+J-1}}\left[\sum_{j=1}^{J^{h}} \sum_{i=1}^{I}\left(G\left(\gamma_{j}\right)\left(z_{j}^{i}\right)-\varepsilon\left(a_{j}\right) z_{j}^{i}-c_{h}\left(a_{j}\right)\right)+w^{h}\right] d \rho\left(\alpha^{n}, A^{n}, \gamma^{n}\right)
$$

converges to

$$
\int_{\Delta^{(L-1) S} \times \Delta^{L+J-1}}\left[\sum_{j=1}^{J^{h}} \sum_{i=1}^{I}\left(G\left(\gamma_{j}\right)\left(z_{j}^{i}\right)-\varepsilon\left(a_{j}\right) z_{j}^{i}-c_{h}\left(a_{j}\right)\right)+w^{h}\right] d \rho(\alpha, A, \gamma),
$$

[see E.4.1 (i)in Mas-Collel [26]]. Then the payoff function of each intermediary is continuous. By assumption (C.1) the type set is compact then we obtain the equicontinuity of payoffs [see Royden [31], chapter 9, problem 40] and the ArzelaAscoli theorem. By assumption (C.2) the game $\mathbb{G}_{I}$ has absolutely continuous information, thus, there exist a distributional strategy equilibrium for the game $\mathbb{G}_{I}$ [see Milgrom and Weber [28], theorem 1].

When $\eta_{h}$ is atomless, the set of pure strategies is dense in the set of distributional strategies [see Milgrom and Weber [28], theorem 3], for each intermediary $h=1, \ldots, H$. This denseness property together with the continuity of the payoffs allow us to conclude that there is a pure strategy profile arbitrarily near any mixed strategy equilibrium which is therefore an $\varepsilon$-equilibrium.

The concept of equilibrium for this economy with incomplete information differs from the previous one precisely in the kind of strategy profile of the first stage game, namely, in this economy with incomplete information the strategies are distributional strategy instead of mixed strategies.

Definition 10. A pair $(\mu, \varphi)$ is a distributional equilibrium for an economy $\mathcal{E}_{I} \equiv\left\{\left(\left(u^{i}, w^{i}\right)_{i \in I},\left(u^{h},\left(T, \eta_{h}\right)\right)_{h \in H}\right)\right\}$, if:

(i) $\mu$ is an equilibrium profile in distributional strategies for the associated game $\mathbb{G}_{I} \equiv\left\{\left(\mathcal{M}_{h}, \pi^{h}\right), h \in H\right\}$ with $\mu=\mu_{1} \times \cdots \times \mu_{H}$.

(ii) $\varphi$ is a measure over prices given by the Gelfand integral of the random selection integrated with respect to the measure $\mu=\mu_{1} \times \cdots \times \mu_{H}$, that is, $\varphi=\rho(\alpha, A, \gamma) d \mu$.

Theorem 2. There is a set $\mathcal{U}^{*} \times \mathcal{W}_{L, \hat{J}}^{*}$ residual in $\mathcal{U} \times \mathbb{R}_{+}^{\hat{J}}$ such that for every economy $\mathcal{E}_{I} \equiv\left\{\left(\left(u^{i}, w^{i}\right)_{i \in I},\left(u^{h},\left(T, \eta_{h}\right)\right)_{h \in H}\right)\right\}$ with $u_{1} \in \mathcal{U}^{*}$ and $w_{L \hat{J}}^{1} \in \mathcal{W}_{L \hat{J}}^{*}$, the distributional equilibrium set is not empty and if in addition $\eta_{h}(h=1, \ldots, H)$ is atomless, then, the economy has an approximate equilibrium in pure strategies.

Proof: The theorem follows from lemma 2 and proposition 17. 


\section{APPENDIX}

Lemma 1. If assumptions A.1. - A.3. and B.1. - B.3. are satisfied, there exist a Walrasian equilibrium for a given vector of spread parameters $\gamma$ and for a given asset return structure $A$.

Proof: Let us consider a truncated economy where commodity plans and portfolios are restricted to lie in a closed cube $K \subseteq \mathbb{R}^{L(S+1)} \times \mathbb{R}^{J}$ with center at the origin. Since a separate budget constraint must be satisfied at every state $s$ and demand is homogenous of degree zero in spot prices, we may restrict our attention to:

$$
\begin{gathered}
p=(\ldots, p(s), \ldots) \in \Delta^{(L-1) S}=\times_{s \in S} \Delta^{L-1} \text { with } \\
\Delta^{L-1}=\left\{p(s) \in \mathbb{R}_{+}^{L}: \sum_{l \in L} p_{l}(s)=1\right\}
\end{gathered}
$$

The homogeneity of degree zero, in prices, of the fist period budget constraint allows us to choose $\left(p_{0}, q\right) \in \Delta^{L+J-1}$ where

$$
\Delta^{L+J-1}=\left\{\left(p_{0}, q\right) \in \mathbb{R}_{+}^{L+J}: \sum_{l=1}^{L} p_{0 l}+\sum_{j=1}^{J} q_{j}=1\right\} .
$$

Now we construct a generalized game played by the $I$ consumers and $S+1$ auctioneers (one for the first period and one for each state of nature of the second period):

(i) For each $i$, consumer $i$ solves:

$$
\begin{array}{cl}
\max _{x \in \mathbb{R}_{+}^{n}, z \in \mathbb{R}^{J}} & u^{i}(x) \\
\text { s.a. } & p_{0}\left(x_{0}-w_{0}^{i}\right)+p_{01} G(\gamma)(z)+q z=0 \\
& p_{s}\left(x_{s}-w_{s}^{i}\right)=p_{1 s} a(s) z \quad \text { for all } \quad(x, z) \in K .
\end{array}
$$

(ii) The first period auctioneer solves:

$$
\begin{aligned}
& \max _{p_{0}, q}\left\{p_{01} \sum_{i=1}^{I}\left(x_{01}^{i}-w_{01}^{i}\right)+p_{01} \sum_{h=1}^{H}\left(x^{h}-w^{h}\right)+p_{01} \sum_{j=1}^{J}\left[\varepsilon\left(a_{j}\right) \sum_{i=1}^{I} z_{+j}^{i}+c\left(a_{j}\right)\right]+\right. \\
& \left.\sum_{l=2}^{L}\left[p_{0 l} \sum_{i=1}^{I}\left(x_{0 l}^{i}-w_{0 l}^{i}\right)\right]+q \sum_{i=1}^{I} z^{i}:\left(p_{0}, q\right) \in \Delta^{L+J-1}\right\} \\
& \text { but since } x^{h}=\sum_{j=1}^{J^{h}} \sum_{i=1}^{I}\left[G\left(\gamma_{j}\right)\left(z_{j}^{i}\right)-\varepsilon\left(a_{j}\right) \bar{z}_{+j}^{i}-c\left(a_{j}\right)\right]+w^{h}, \text { we can rewrite }
\end{aligned}
$$


the problem:

$\max _{p_{0}, q}\left\{p_{01} \sum_{i=1}^{I}\left(x_{01}^{i}-w_{01}^{i}\right)+p_{01} \sum_{h=1}^{H}\left(\left[\sum_{j=1}^{J^{h}} \sum_{i=1}^{I}\left[G\left(\gamma_{j}\right)\left(z_{j}^{i}\right)-\varepsilon\left(a_{j}\right) z_{+j}^{i}-c\left(a_{j}\right)\right]+\right.\right.\right.$
$\left.\left.w^{h}\right]-w^{h}\right)+p_{01} \sum_{j=1}^{J}\left[\varepsilon\left(a_{j}\right)\left(\sum_{i=1}^{I} z_{+j}^{i}\right)+c\left(a_{j}\right)\right]+\sum_{l=2}^{L}\left[p_{0 l} \sum_{i=1}^{I}\left(x_{0 l}^{i}-w_{0 l}^{i}\right)\right]+q \sum_{i=1}^{I} z^{i}:$
$\left.\left(p_{0}, q\right) \in \Delta^{L+J-1}\right\} \Longleftrightarrow$ $\max _{p_{0}, q}\left\{p_{01} \sum_{i=1}^{I}\left(x_{01}^{i}-w_{01}^{i}\right)+p_{01} \sum_{h=1}^{H}\left(\left[\sum_{j=1}^{J^{h}} \sum_{i=1}^{I} G\left(\gamma_{j}\right)\left(z_{j}^{i}\right)\right]+\sum_{l=2}^{L}\left[p_{0 l} \sum_{i=1}^{I}\left(x_{0 l}^{i}-w_{0 l}^{i}\right)\right]+\right.\right.$ $\left.q \sum_{i=1}^{I} z^{i}:\left(p_{0}, q\right) \in \Delta^{L+J-1}\right\} \Longleftrightarrow$ $\max _{p_{0}, q}\left\{p_{01} \sum_{i=1}^{I}\left(x_{01}^{i}-w_{01}^{i}\right)+p_{01} \sum_{i=1}^{I} \sum_{h=1}^{H}\left(\left[\sum_{j=1}^{J^{h}} G\left(\gamma_{j}\right)\left(z_{j}^{i}\right)\right]+\sum_{l=2}^{L}\left[p_{0 l} \sum_{i=1}^{I}\left(x_{0 l}^{i}-w_{0 l}^{i}\right)\right]+\right.\right.$ $\left.q \sum_{i=1}^{I} z^{i}:\left(p_{0}, q\right) \in \Delta^{L+J-1}\right\} \Longleftrightarrow$ $\max _{p_{0}, q}\left\{p_{01} \sum_{i=1}^{I}\left(x_{01}^{i}-w_{01}^{i}\right)+p_{01} \sum_{i=1}^{I} \sum_{j=1}^{J} G\left(\gamma_{j}\right)\left(z_{j}^{i}\right)+\sum_{l=2}^{L}\left[p_{0 l} \sum_{i=1}^{I}\left(x_{0 l}^{i}-w_{0 l}^{i}\right)\right]+\right.$ $\left.q \sum_{i=1}^{I} z^{i}:\left(p_{0}, q\right) \in \Delta^{L+J-1}\right\} \Longleftrightarrow$ $\max _{p_{0}, q}\left\{p_{01} \sum_{i=1}^{I}\left(x_{01}^{i}+G(\gamma)\left(z^{i}\right)-w_{01}^{i}\right)+\sum_{l=2}^{L}\left[p_{0 l} \sum_{i=1}^{I}\left(x_{0 l}^{i}-w_{0 l}^{i}\right)\right]+q \sum_{i=1}^{I} z^{i}:\left(p_{0}, q\right) \in\right.$ $\left.\Delta^{L+J-1}\right\}$.

(iii) The second period auctioneers solves:

$$
\max _{p_{s}}\left\{p_{s} \cdot \sum_{i=1}^{I}\left(x_{s}^{i}-w_{s}^{i}\right): p_{s} \in \Delta^{L-1}\right\} .
$$

This generalized game satisfies the assumptions of Debreu on existence of strategic equilibria. Let $\left((\bar{x}, \bar{z}), \bar{p}_{0}, \bar{p}_{1}, \bar{q}\right)$ be a strategic equilibrium. The first period budget constraint of each consumer implies,

$$
\begin{aligned}
& p_{01} \sum_{i=1}^{I}\left(x_{01}^{i}+G(\gamma)\left(z^{i}\right)-w_{01}^{i}\right)+\sum_{l=2}^{L}\left[p_{0 l} \sum_{i=1}^{I}\left(x_{0 l}^{i}-w_{0 l}^{i}\right)\right]+q \sum_{i=1}^{I} z^{i} \leq 0 . \\
& \text { Then, by (ii), } \sum_{i=1}^{I}\left(\bar{x}_{01}^{i}+G(\gamma)\left(\bar{z}^{i}\right)-w_{01}^{i}\right) \leq 0 \text { and } \sum_{i=1}^{I}\left(\bar{x}_{0 l}^{i}-w_{0 l}^{i}\right) \leq 0, \text { for all }
\end{aligned}
$$


$l=2, \ldots, L$. Therefore, $\left.\sum_{i=1}^{I} \bar{x}_{01}^{i} \leq \sum_{i=1}^{I}\left[w_{01}^{i}-G(\gamma)\left(\bar{z}^{i}\right)\right]\right) \leq \sum_{i=1}^{I} w_{01}^{i}$, since $G(\gamma)>$ 0. Thus, in equilibrium, the consumption bundle of the first period satisfies, $\sum_{i=1}^{I} \bar{x}_{01}^{i} \leq \sum_{i=1}^{I} w_{01}^{i}$ and $\sum_{i=1}^{I} \bar{x}_{0 l}^{i} \leq \sum_{i=1}^{I} w_{0 l}^{i}$, for all $l=2, \ldots, L$.

By (ii) we also have $\sum_{i=1}^{I} \bar{z}^{i} \leq 0$, implying, $A_{s} \sum_{i=1}^{I} \bar{z}^{i} \leq 0$. Then, $p_{s} \cdot \sum_{i=1}^{I}\left(\bar{x}_{s}^{i}-\right.$ $\left.w_{s}^{i}\right) \leq 0$, for each $s$, and, therefore, by (iii), $\sum_{i=1}^{I}\left(\bar{x}_{s}^{i}-w_{s}^{i}\right) \leq 0$, for each $s$. The equilibrium consumption bundle of the second period satisfies $\sum_{i=1}^{I} \bar{x}_{s}^{i} \leq \sum_{i=1}^{I} w_{s}^{i}$, for all $s \in S$.

Now, consider a sequence of increasing closed cubes $K^{n}$ whose centers are the origin and let $\left(\bar{x}^{n}, \bar{z}^{n}, \bar{p}_{0}^{n}, \bar{p}_{1}^{n}, \bar{q}^{n}\right)$ be a respective sequence of strategic equilibria for the associated truncated generalized games. Notice that $\bar{x}^{n} \in\left[0, \sum_{i=1}^{I} w^{i}\right]$, $\bar{p}_{1}^{n} \in \Delta^{(L-1) S}$ and $\left(\bar{p}_{0}^{n}, \bar{q}^{n}\right) \in \Delta^{L+J-1}$ and therefore there exists a converging subsequence $\left(\bar{x}, \bar{p}_{0}, \bar{p}_{1}, \bar{q}\right)^{n_{q}}$ whose limit is, say, $\left(\tilde{x}, \tilde{p}_{0}, \tilde{p}_{1}, \tilde{q}\right) \in\left[0, \sum_{i=1}^{I} w^{i}\right] \times \Delta^{(L-1) S} \times$ $\Delta^{L+J-1}$.

If $\tilde{p}_{1 s}>0$ for all $s$, then by the first order necessary and sufficient conditions for the consumer problem we obtain $\tilde{z}^{i}$. Now, $\tilde{z}^{i}=\lim _{n_{q}} \bar{z}^{n_{q}}$ (by continuity of the first order conditions) and all other budget constraints will be satisfied, together with the inequalities $\sum_{i=1}^{I}\left(\tilde{x}_{01}^{i}+G(\gamma)\left(\tilde{z}^{i}\right)-w_{01}^{i}\right) \leq 0, \sum_{i=1}^{I}\left(\tilde{x}_{0 l}^{i}-w_{0 l}^{i}\right) \leq 0$, for all $l=2, \ldots, L, \sum_{i=1}^{I}\left(\tilde{x}_{s}^{i}-w_{s}^{i}\right) \leq 0$ for all $s$ and $\sum_{i=1}^{I} \tilde{z}^{i} \leq 0$.

Now we prove that $\left(\tilde{x}^{i}, \tilde{z}^{i}\right)$ is an optimal choice for consumer $i$ at prices $\left(\tilde{p}_{0}, \tilde{p}_{1}, \tilde{q}\right)$. Suppose it were not, say $\left(\hat{x}^{i}, \hat{z}^{i}\right)$ is budget feasible at $\left(\tilde{p}_{0}, \tilde{p}_{1}, \tilde{q}\right)$ and $u^{i}\left(\hat{x}^{i}\right)>u^{i}\left(\tilde{x}^{i}\right)$. For $n$ large enough, $\left(\tilde{x}^{i}, \tilde{z}^{i}\right) \in \operatorname{int} K^{n}$ and for $\lambda$ sufficiently close to zero we have $\lambda\left(\hat{x}^{i}, \hat{z}^{i}\right)+(1-\lambda)\left(\tilde{x}^{i}, \tilde{z}^{i}\right)$ belonging to $K^{n}$, budget feasible at $\left(\tilde{p}_{0}, \tilde{p}_{1}, \tilde{q}\right)$ and such that $u^{i}\left(\lambda \hat{x}^{i}+(1-\lambda) \tilde{x}^{i}\right)>u^{i}\left(\tilde{x}^{i}\right)$. By continuity of preferences, $\left(\lambda \hat{x}^{i}+(1-\lambda) \bar{x}^{i n_{q}}, \lambda \hat{z}^{i}+(1-\lambda) \bar{z}^{i n_{q}}\right)$ would be chosen instead of $\left(\bar{x}^{i n_{q}}, \bar{z}^{i n_{q}}\right)$, in the truncated economy $K^{n}$, at prices $\left(\bar{p}_{0}^{n_{q}}, \bar{p}_{1}^{n_{q}}, \bar{q}^{n_{q}}\right)$, a contradiction. 
Let us now prove that $\sum_{i=1}^{I} \tilde{z}^{i}=0, \sum_{i=1}^{I}\left(\tilde{x}_{01}^{i}+G(\gamma)\left(\tilde{z}^{i}\right)-w_{01}^{i}\right)=0, \sum_{i=1}^{I}\left(\tilde{x}_{0 l}^{i}-\right.$ $\left.w_{0 l}^{i}\right)=0$, for all $l=2, \ldots, L$ and $\sum_{i=1}^{I}\left(\tilde{x}_{s}^{i}-w_{s}^{i}\right)=0$ for all $s$.

The first order conditions of the optimization problem of the period-0 auctioneer imply that $\sum_{i=1}^{I} z^{i}=\beta=\sum_{i=1}^{I}\left(x_{01}^{i}+G(\gamma)\left(z^{i}\right)-w_{01}^{i}\right)=\sum_{i=1}^{I}\left(x_{0 l}^{i}-w_{0 l}^{i}\right)$, for any $l$, where $\beta$ is the Lagrange multiplier for the constraint $\sum_{l=1}^{L} p_{0 l}+\sum_{j=1}^{J} q_{j}=1$. Then, by Walras' law, $p_{01} \sum_{i=1}^{I}\left(x_{01}^{i}+G(\gamma)\left(z^{i}\right)-w_{01}^{i}\right)+\sum_{l=1}^{L}\left[p_{0 l} \sum_{i=1}^{I}\left(x_{0 l}^{i}-w_{0 l}^{i}\right)\right]+q \cdot \sum_{i=1}^{I} z^{i}=$ $p_{01} \beta+\sum_{l=1}^{L}\left[p_{0 l} \beta\right]+q \cdot \beta=0$, that is, $\beta\left[\sum_{l=1}^{L} p_{0 l}+\sum_{j=1}^{J} q_{j}\right]=1$. But $\sum_{l=1}^{L} p_{0 l}+\sum_{j=1}^{J} q_{j}=1$ which implies $\beta=0$ and therefore there is no free disposal. Finally, since $\sum_{i=1}^{I} \tilde{z}^{i}=0$, then by budget constraint $p_{s} \cdot \sum_{i=1}^{I}\left(\tilde{x}_{s}^{i}-w_{s}^{i}\right)=0$, and we have no free disposal as well.

It remains to check that $\tilde{p}_{1 s} \neq 0$ for any $s$. Suppose $\tilde{p}_{1 s}=0$ for some $s$. Let $e_{1 s}$ be a canonical vector, in the direction of this commodity. By the monotonicity assumption, $u^{i}\left(\tilde{x}^{i}+k e_{1 s}\right)>u^{i}\left(\tilde{x}^{i}\right)$, for $k>0$. Let $k=\min _{l} w_{s l}^{i}$, then, for $\mathrm{n}$ large enough, $u^{i}\left(\left(1-\bar{p}_{1 s n}^{n_{q}}\right) \tilde{x}^{i}+k e_{1 s}\right)>u^{i}\left(\tilde{x}^{i n_{q}}\right)$. But it is easily seen that the consumer can afford the bundle on the left hand side (at prices $\left(\bar{p}_{0}^{n_{q}}, \bar{p}_{1}^{n_{q}}, \bar{q}^{n_{q}}\right)$ ), by choosing the portfolio $\left(1-\bar{p}_{1 s}\right)^{n_{q}} \bar{z}^{i n_{q}}$. This contradicts the fact that $\left(\bar{x}, \bar{z}, \bar{p}_{0}, \bar{p}_{1}, \bar{q}\right)^{n_{q}}$ is a free disposal equilibrium for the truncated economy with cube $K^{n_{q}}$.

Lemma 2. If $(A .1)-(A .3)$ and $(B .1)-(B .3)$ are satisfied, individual demand is a $C^{1}$ function.

Proof: Let us denote by $x_{0}$ the consumers consumption vector of the first period and by $x_{1}$ the consumers consumption vector of the second period. Let us fix $p_{s 1}=1$, for any $s$. The first order necessary and sufficient conditions for the consumer problem are: 


$$
\begin{aligned}
& D_{0} u^{i}-\mu p_{0}=0 \\
& D_{s} u^{i}-\lambda_{s} p_{s}=0, s=1, \ldots, S \\
& -p_{s} \cdot\left(x_{s}-w_{s}^{i}\right)+A_{s} z=0, s=1, \ldots, S \\
& \lambda^{\prime} A-\mu\left[q+D_{z} G\right]=0 \\
& -p_{0}\left(x_{0}-w_{0}^{i}\right)-q . z-p_{01} G(z)=0
\end{aligned}
$$

Let $\mathbf{J}$ be the $S L \cdot S$ matrix where the $s^{t h}$ column is $\left.\left(0 \ldots 0 p_{s}^{\prime} 0 \ldots 0\right)\right)$. The Jacobian matrix with the second order derivatives(with respect to $\left(x_{0}, x_{1}, \lambda, z, \mu\right)$ is:

$$
\mathbf{J}=\left[\begin{array}{ccccc}
D_{0}^{2} u^{i} & 0 & 0 & 0 & -p_{0} \\
0 & D_{1}^{2} u^{i} & -p_{1} & 0 & 0 \\
0 & -p_{1}^{\prime} & 0 & A & 0 \\
0 & 0 & A^{\prime} & -\mu D_{z}^{2} & -q-D_{z} G \\
-p_{0} & 0 & 0 & -q^{\prime}-D_{z}^{\prime} G & 0
\end{array}\right]
$$

It is easy to observe that the matrix $\mathbf{J}$ is non-singular. In fact, let $y=\left(\hat{x}_{0}, \hat{x}_{1}, \hat{\lambda}, \hat{z}, \hat{\mu}\right)$ such that $\mathbf{J} y=0$, then $y^{\prime} \mathbf{J} y=0$ (which using $\mathbf{J} y=0$ ) reduces to $\hat{x}_{0}^{\prime}\left(D_{0}^{2} u^{i}\right) \hat{x}_{0}+$ $\hat{x}_{1}^{\prime}\left(D_{1}^{2} u^{i}\right) \hat{x}_{1}-\hat{z}^{\prime}\left(\mu D_{z}^{2} G\right) \hat{z}$. Notice that this last equality can be written as

$$
\left[\begin{array}{lll}
\hat{x}_{0}^{\prime} & \hat{x}_{1}^{\prime} & \hat{z}
\end{array}\right]\left[\begin{array}{ccc}
D_{0}^{2} u^{i} & 0 & 0 \\
0 & D_{1}^{2} u^{i} & 0 \\
0 & 0 & -\mu D_{z}^{2} G
\end{array}\right]\left[\begin{array}{c}
\hat{x}_{0} \\
\hat{x}_{1} \\
\hat{z}
\end{array}\right]
$$

which implies $\hat{x}_{0}=0, \hat{x}_{1}=0$ and $\hat{z}=0$ by negative definiteness of $D^{2} u^{i}$ and $\mu D_{z}^{2} G$. Then, back to $\mathbf{J} y=0$, we get $\hat{\lambda}=0$. Finally again with $\mathbf{J} y=0, \hat{\mu}=0$. Therefore by the implicit function theorem we conclude that individual excess demand is a $C^{1}$ function.

Proposition 1. There is a set $\mathcal{U}^{*} \times \mathcal{W}_{L, \hat{J}}^{*}$ residual in $\mathcal{U} \times \mathbb{R}_{+}^{\hat{J}}$ such that for an economy $\left\{\left(u^{i}\right)_{i \in I},\left(w_{L \hat{J}}^{1}, w_{-L \hat{J}}^{1}\right),\left(u^{h}, w^{h}\right)_{h \in H}, \gamma, A\right\}$ with $u_{1} \in \mathcal{U}^{*}, w_{L \hat{J}}^{1} \in \mathcal{W}_{L \hat{J}}^{*}$ and with an exogenous vector of spread parameters $\gamma \in \Gamma^{J}$ and an exogenous asset market given by the payoff matrix $A \in \mathcal{A}^{J}$ the number of critical equilibria for the economy is at most $n I+S J+J$.

The proof requires some notation and a preparatory Lemma.

Let $G$ be a closed ball in $\Gamma^{J}$ with rational center and rational coordinates and let $M$ be a closed ball in $\mathcal{A}^{J}$ with rational center and rational coordinates. Let $B \times D=\left\{B^{1} \times D^{1}, \ldots, B^{n I+S J+J} \times D^{n I+S J+J}\right\}$ be a collection of disjoint 
closed balls in $\Delta^{(L-1) S} \times \Delta^{L+J-1}$, each $B^{i} \times D^{i}$ having rational radius and a center with rational first $(L-1)(S+1)+J$ coordinates. Now, for every $B$ and positive integer $m$ put $K_{B, m}=\left\{w \in \mathbb{R}_{+}^{L(1+S-\hat{J})+(L-1) \hat{J}+n(I-1)}:(1 / m) \leq p^{j} \cdot w^{i} \leq\right.$ $m$, for all $j, i$ and for all $\left.p^{j} \in B^{j}\right\}$. There is a countable number of product sets $K_{B, m} \times G \times M \times B \times D$.

For every $u_{1} \in \mathcal{U}$ and every $w_{L \hat{J}}^{1}$ define

$$
F_{\left(u_{1}, w_{L \hat{J}}^{1}\right)}: K_{B, m} \times G \times M \times B \times D \rightarrow \mathbb{R}^{((L-1)(S+1)+\hat{J})(n I+S J+J+1)}
$$

with $F_{\left(u_{1}, w_{L, \hat{J}}^{1}\right)}^{j}\left(w_{-L \hat{J}}, \gamma, A,\left(p_{0}^{1}, p_{1}^{1}, q^{1}\right), \ldots,\left(p_{0}^{n I+S J+J+1}, p_{1}^{n I+S J+J+1}, q^{n I+S J+J+1}\right)\right)$, $j=1, \ldots, n I+S J+J+1$, defined as follows: the first $(L-1)(S+1)$ coordinates comprise the aggregate excess demand function for the first $L-1$ commodities on period 1 and for the first $L-1$ commodities on each state of period 2, the last $\hat{J}$ components of this function consist of aggregate demand for assets 1 to $J$ summed up in the compound portfolio $\hat{z}=\hat{z}^{1}+B \hat{z}^{2}$. These commodities and portfolio demand are those generated by the economy $\left\{\left(u^{i}\right)_{i \in I},\left(w_{L \hat{J}}^{1}, w_{-L \hat{J}}^{1}\right),\left(u^{h}, w^{h}\right)_{h \in H}, \gamma, A\right\}$ at the price vector $\left(p_{0}^{j}, p_{1}^{j}, q^{j}\right)$.

Lemma 3. There is an open and dense subset $\mathcal{U}^{\prime} \times \mathcal{W}_{L \hat{J}}^{\prime} \subset \mathcal{U} \times \mathbb{R}_{+}^{\hat{J}}$ such that for every element $u_{1}$ belonging to $\mathcal{U}^{\prime}$ and every $w_{L \hat{J}}^{1}$ belonging to $\mathcal{W}_{L \hat{J}}^{\prime}, 0$ is a regular value of $F_{\left(u_{1}, w_{L \hat{\jmath}}^{1}\right)}$.

Proof: Openness follows from continuity of the determinant function and compactness of the sets $K_{B, m} \times G \times M \times B \times D$ (because $F_{\left(u_{1}, w_{L, j}^{1}\right)}$ turns into an open map). So we only need to concern about density, that is, given $\left(u_{1}, w_{L \hat{J}}^{1}\right)$ we have to show that there exists $\left(u_{1}^{\prime}, w_{L \hat{J}}^{\prime 1}\right)$ arbitrarily near from $\left(u_{1}, w_{L \hat{J}}^{1}\right)$ such that 0 is a regular value of $F_{\left(u_{1}^{\prime}, w_{L \hat{J}}^{\prime 1}\right.}$.

Let $G^{\prime}$ such that $G^{\prime} \subset \operatorname{int} G$. Let $M^{\prime}$ such that $M^{\prime} \subset$ int $M$. Let $B^{\prime} \times D^{\prime}$ be a collection (in the countable family) with $B^{\prime j} \times D^{\prime j} \subset$ int $B^{j} \times$ int $D^{j}$ for every $j$. Let also $\alpha_{j}: \Gamma \times \mathcal{A} \times \Delta^{(L-1) S} \times \Delta^{L+J-1} \times \mathbb{R}^{S+1} \rightarrow[0,1]$ be a $C^{\infty}$ function satisfying $\alpha_{j}\left(G^{\prime} \times M^{\prime} \times B^{\prime j} \times D^{\prime j} \times[1 / m, m]^{S+1}\right)=1$ and

$\alpha_{j}\left(\Gamma \times \mathcal{A} \times \Delta^{(L-1) S} \times \Delta^{L+J-1} \times \mathbb{R} \backslash G \times M \times B^{j} \times D^{j} \times[2 / m, 2 m]^{S+1}\right)=0$ Now fix $\varepsilon>0$ and let $v: \Gamma \times \mathcal{A} \times \Delta^{(L-1) S} \times \Delta^{L+J-1} \times \mathbb{R}^{S+1} \rightarrow \mathbb{R}$ be the indirect utility function associated with $u_{1}$. For every $j$ denote by $\mathcal{Y}^{j} \subset \mathbb{R}^{(S+1)(L-1)}$ and by $\mathcal{T}^{j} \subset \mathbb{R}^{\hat{J}}$ the corresponding unit balls. Then for every $(y, t) \in \mathcal{Y}^{1} \times \ldots \times$ $\mathcal{Y}^{n I+S J+J+1} \times \mathcal{T}^{1} \times \ldots \times \mathcal{T}^{n I+S J+J+1}$, we define 


$$
\begin{aligned}
& v(y, t)\left(\gamma, A, p_{0}, p_{1}, q, N\right)=v\left(\gamma, A, p_{0}, p_{1}, q, N_{t}\right)+ \\
& \varepsilon \sum_{j=1}^{n I+S J+J+1} \alpha_{j}\left(\gamma, A, p_{0}, p_{1}, q, N\right) y^{j} \cdot\left(p_{0}, p_{1}\right)
\end{aligned}
$$

where $N=\left(p_{0}, p_{1}\right) \cdot\left(w_{0}^{1}, w_{1}^{1}\right)$ and

$N_{t}=N+\varepsilon \sum_{j=1}^{n I+S J+J+1} \alpha_{j}\left(\gamma, A, p_{0}, p_{1}, q, N\right) \sum_{k=1}^{\hat{J}} p_{k L} t_{k}^{j}$

Notice that, by Cass trick [ Geanakoplos [18] or Magill and Shafer[25]] we can consider, without loss of generality, that first consumer's problem is a solution of a problem with complete markets. Thus, by duality theory (see, for example, Diewert (1982)), we obtain that if $\varepsilon$ is sufficiently small, then for all $(y, t), v_{(y, t)}: \Gamma \times \mathcal{A} \times \Delta^{(L-1) S} \times \Delta^{L+J-1} \times \mathbb{R}^{S+1} \rightarrow \mathbb{R}$ satisfies all the properties of an indirect utility function. If $u_{1}^{y}$ is the corresponding direct utility function, then by taking $\varepsilon$ arbitrarily small, $u_{1}^{y}$ is the perturbed utility function and $w_{t}^{1}=w_{L \hat{J}}^{1}+\varepsilon \sum_{j=1}^{n I+S J+J+1} \alpha_{j}\left(\gamma, A, p_{0}, p_{1}, q, N\right) t^{j}$ is the corresponding initial endowment that we are looking for. Thus, the lemma is proved if we show that for any $\varepsilon>0$, there exists $(y, t)$ such that $F_{\left(u_{1}^{y}, w_{t}^{1}\right)}$ is transverse to 0 . In order to obtain the result, we define

$F: K_{B, m} \times G \times M \times\left(B^{k} \times D^{k} \times \mathcal{Y}^{k} \times \mathcal{T}^{k}\right)_{k=1}^{k=n I+S J+J+1} \rightarrow \mathbb{R}^{(n I+S J+J+1)((L-1)(S+1)+\hat{J})}$ by, $F^{j}\left(w, \gamma, A,\left(p_{0}^{1}, p_{1}^{1}, q^{1}\right), \ldots,\left(p_{0}^{n I+S J+J+1}\right.\right.$, such that as before, the first $(L-$ 1) $(S+1)$ coordinates comprise the aggregate excess demand function for the first $L-1$ commodities on period 1 and for the first $L-1$ commodities on each state of period 2 and the last $\hat{J}$ components of this function consist of aggregate demand for assets summed up in the compound portfolio $\hat{z}=\hat{z}^{1}+B \hat{z}^{2}$. We are taking up the demand generated by the perturbed economy $\left(u_{1}^{y}, w_{t}^{1}\right)$, at the price vector $\left(p_{0}^{j}, p_{1}^{j}, q^{j}\right)$. To obtain the result let us show that rank

$$
\begin{gathered}
D_{(y, t)} F\left(\bar{w}, \bar{\gamma}, \bar{A},\left(\bar{p}_{0}^{1}, \bar{p}_{1}^{1}, \bar{q}^{1}\right), \ldots,\left(\bar{p}_{0}^{n I+S J+J+1}, \bar{p}_{1}^{n I+S J+J+1}, \bar{q}^{n I+S J+J+1}\right), \bar{y}, \bar{t}\right) \\
=(n I+S J+J+1)((L-1)(S+1)+\hat{J})
\end{gathered}
$$

whenever $F\left(\bar{w}, \bar{\gamma}, \bar{A},\left(\bar{p}_{0}^{1}, \bar{p}_{1}^{1}, \bar{q}^{1}\right), \ldots,\left(\bar{p}_{0}^{n I+S J+J+1}, \bar{p}_{1}^{n I+S J+J+1}, \bar{q}^{n I+S J+J+1}\right), \bar{y}, \bar{t}\right)=$ 0. Thus, by Transversality Theorem [see Guillemin and Pollack (1994)], one verifies that for almost all $y, 0$ is a regular value of $F_{u_{1}^{y}}$. Note that by construction 
$D_{(y, t)} F\left(\bar{w}, \bar{\gamma}, \bar{A},\left(\bar{p}_{0}^{1}, \bar{p}_{1}^{1}, \bar{q}^{1}\right), \ldots,\left(\bar{p}_{0}^{n I+S J+J+1}, \bar{p}_{1}^{n I+S J+J+1}, \bar{q}^{n I+S J+J+1}\right), \bar{y}, \bar{t}\right)$ is diagonal (i.e., $D_{\left(y^{j^{\prime}, t j^{\prime \prime}}\right)} F^{j}=0$ for every $\left.j \neq j^{\prime}, j^{\prime \prime}\right)$, therefore, it suffices to show that the rank of the matrix

$$
D_{(y, t)} F^{j}\left(\bar{w}, \bar{\gamma}, \bar{A},\left(\bar{p}_{0}^{1}, \bar{p}_{1}^{1}, \bar{q}^{1}\right), \ldots,\left(\bar{p}_{0}^{n I+S J+J+1}, \bar{p}_{1}^{n I+S J+J+1}, \bar{q}^{n I+S J+J+1}\right), \bar{y}, \bar{t}\right)
$$
is $((L-1)(S+1)+\hat{J})$.

To calculate this rank, remember that $\left(y^{j}, t^{j}\right)$ enters into $F^{j}$ only via the excess demand for the first consumer. Moreover, taking into account the Cass trick [see Geanakoplos (1990) or Magill and Shafer (1991)] the demand of first consumer can be computed as $\underset{x}{\operatorname{argmax}}\left\{u_{1}(x): x \in B^{1}\left(\tilde{p}_{0}, \tilde{p}_{1}\right)\right\}$ with

$$
\left.B^{1}\left(\tilde{p}_{0}, \tilde{p}_{1}\right)\right\}=\left\{x \in \mathbb{R}^{L(S+1)}: \tilde{p}_{0} x_{0}+\tilde{p}_{1} x_{1} \leq \tilde{p}_{0} w_{0}^{1}+\tilde{p}_{1} w_{1}^{1}\right\} \text {, where }\left(\tilde{p}_{0}, \tilde{p}_{1}\right)
$$

is equal to $\left(p_{0}, p_{1}\right)$ rescaled, that is, the demand of the first consumer is a solution of a problem with complete markets, so we can apply the Roy' formula. Then the excess demand for commodities of the first consumer is given by

$$
-\left[\frac{1}{D_{w} v_{y}\left(\bar{p}_{0}^{j}, \bar{p}_{1}^{j}, \bar{p}_{0}^{j} \bar{w}_{0}^{1}+\bar{p}_{1}^{j} \bar{w}_{1}^{1}\right)}\right] D_{\left(p_{0}, p_{1}\right)} v_{y}\left(\bar{p}_{0}^{j}, \bar{p}_{1}^{j}, \bar{p}_{0}^{j} \bar{w}_{0}^{1}+\bar{p}_{1}^{j} \bar{w}_{1}^{1}\right)-\bar{w}_{0}-\bar{w}_{1} .
$$

Notice that $D_{w} v_{y}\left(\bar{p}_{0}^{j}, \bar{p}_{1}^{j}, \bar{p}_{0}^{j} \bar{w}_{0}^{1}+\bar{p}_{1}^{j} \bar{w}_{1}^{1}\right)$ does not depend on $z^{j}$ and by definition of $v_{y}$ we have that

$$
D_{\left(p_{0}, p_{1}\right)} v_{y}\left(\bar{p}_{0}^{j}, \bar{p}_{1}^{j}, \bar{p}_{0}^{j} \bar{w}_{0}^{1}+\bar{p}_{1}^{j} \bar{w}_{1}^{1}\right)=D_{\left(p_{0}, p_{1}\right)} v\left(\bar{p}_{0}^{j}, \bar{p}_{1}^{j}, \bar{p}_{0}^{j} \bar{w}_{0}^{1}+\bar{p}_{1}^{j} \bar{w}_{1}^{1}\right)+\varepsilon y^{j}, \text { a linear }
$$
map on $y^{j}$.

By inversion of a Cramer subsystem of budget equations for consumer 1 we can obtain that the compound portfolio of assets for this consumer 1 is given by

$$
\hat{z}^{1}=\hat{A}^{\prime-1}\left[p_{s} \cdot\left(x_{s}-w_{s}^{1}\right)\right]_{s=1}^{S},
$$

Where $\hat{A}^{\prime}$ is a non-singular $\hat{J} \times \hat{J}$ submatrix of $\hat{A}$. Since $\hat{A}$ is in general position, any $\hat{J} \times \hat{J}$ submatrix of $\hat{A}$ is non-singular and to simplify let us take the matrix formed by the first $\hat{J}$ rows. Then the compound portfolio demand $\hat{z}$ is a linear map on the demand for commodities. So, the compound portfolio demand $\hat{z}$ is also a linear map on $y^{j}$.

Now, we can write $D_{\left(y^{j}, t^{j}\right)} F^{j}$ as follows:

$$
\begin{aligned}
& D_{(y, t)} F^{j}=\left(\begin{array}{ccc}
\mathcal{F}_{11} & \vdots & \mathcal{F}_{12} \\
\ldots & \ldots \\
\mathcal{F}_{21} & \vdots & \mathcal{F}_{22}
\end{array}\right) \text { where } \\
& \mathcal{F}_{11}=\frac{\partial x^{1}}{\partial y^{j}}, \quad \mathcal{F}_{12}=\frac{\partial x^{1}}{\partial t^{j}}, \quad \mathcal{F}_{21}=\frac{\partial \tilde{z}^{1}}{\partial y^{j}}, \quad \mathcal{F}_{22}=\frac{\partial \tilde{z}^{1}}{\partial t^{j}} .
\end{aligned}
$$


Note that $\left|D_{(y, t)} F^{j}\right|=\left|\mathcal{F}_{11}\right| \cdot\left|\mathcal{F}_{22}-\mathcal{F}_{21} \cdot \mathcal{F}_{11}^{-1} \cdot \mathcal{F}_{12}\right|$.

By the Roy's formula above, $\mathcal{F}_{11}=\varepsilon I$, where $I$ is the unit matrix of order $(S+1)(L-1)$.

On the other hand, the line $k$ of the matrix $\mathcal{F}_{22}$ and the line $k$ of the matrix $\mathcal{F}_{21}$ are defined as follows:

$$
\begin{aligned}
\left(\mathcal{F}_{22}\right)_{k} & =\left(\hat{A}^{\prime-1}\right)_{k}\left(\sum_{l} p_{s l} \frac{\partial x_{s l}^{1}}{\partial t_{k}^{j}}\right)_{s=1}^{J^{\prime}}-\varepsilon\left(\hat{A}^{\prime-1}\right)_{k} \\
\left(\mathcal{F}_{21}\right)_{k} & =\left(\hat{A}^{\prime-1}\right)_{k}\left(\sum_{l} p_{s l} \frac{\partial x_{s l}^{1}}{\partial y_{k}^{j}}\right)_{s=1}^{J^{\prime}}
\end{aligned}
$$

Then, the determinant of $D_{\left(y^{j}, t^{j}\right)} F^{j}$ is given by

$$
\left|D_{\left(y^{j}, t^{j}\right)} F^{j}\right|=\varepsilon\left|\left(\hat{A}^{\prime-1}\right)\left(\sum_{l} p_{s l} \frac{\partial x^{1}}{\partial t^{j}}\right)-\varepsilon\left(\hat{A}^{\prime-1}\right)-\varepsilon^{-1} \hat{A}^{\prime-1}\left(\sum_{l} p_{s l} \frac{\partial x^{1}}{\partial y^{j}}\right) \frac{\partial x}{\partial t^{j}}\right| .
$$

Summing up, we obtain that $\left|D_{\left(y^{j}, t^{j}\right)} F^{j}\right|=-\varepsilon^{2}\left|\hat{A}^{\prime-1}\right|$. Since, $\hat{A}^{\prime-1}$ is a nonsingular matrix, the determinant of $D_{\left(y^{j}, t^{j}\right)} F^{j}$ is non-null. Finally, $D_{\left(y^{j}, t^{j}\right)} F^{j}$ has $\operatorname{rank}(L-1)(S+1)+\hat{J}$.

Proof: (Proof of Proposition 1.) By lemma 3 we know that there is an open and dense set $\mathcal{U}^{\prime} \times \mathcal{W}_{L \hat{J}}^{\prime} \subset \mathcal{U} \times \mathbb{R}_{+}^{\hat{J}}$ such that for every $\left(u_{1}, w_{L \hat{J}}^{1}\right) \in \mathcal{U}^{\prime} \times \mathcal{W}_{L \hat{J}}^{\prime}$, 0 is a regular value of $F_{\left(u_{1}, w_{L \hat{\jmath}}^{1}\right)}$. Consider the intersection $\mathcal{U}^{*} \times \mathcal{W}_{L \hat{J}}^{*}$ of the sets $\mathcal{U}^{\prime} \times \mathcal{W}_{L \hat{J}}^{\prime}$ obtained as we run over all the different $G, M, B \times D$ and $m$. This set is clearly residual. Suppose now that for some $u_{1} \in \mathcal{U}^{*}, \bar{w}_{L \hat{J}}^{1} \in \mathbb{R}^{n I}$ with $\bar{w}_{L \hat{J}}^{1} \in \mathcal{W}_{L \hat{J}}^{*}, \gamma \in \Gamma$ and $\bar{A} \in \mathcal{A}$ we have $n I+S J+J+1$ critical equilibria, $\left(\bar{p}_{0}^{1}, \bar{p}_{1}^{1}, \bar{q}^{1}\right), \ldots,\left(\bar{p}_{0}^{n I+S J+J+1}, \bar{p}_{1}^{n I+S J+J+1}, \bar{q}^{n I+S J+J+1}\right)$. Consider $\gamma$ such that $\gamma \in$ $G$, consider $M$ such that $A \in M, B \times D$ and $m$ such that each $B^{j} \times D^{j}$ contains $\left({\overline{p_{0}}}^{j},{\overline{p_{1}}}^{j}, \bar{q}^{j}\right)$ in its interior and $\bar{w}_{-L \hat{J}}$ belongs to $K_{B, m}$. Now let $F_{u_{1}, w_{L \hat{J}}^{1}}$ defined on this $G, M, B \times D$ and $K_{B, m}$. We have that rank of $D_{\gamma} F_{\left(u_{1}, w_{L \hat{J}}^{1}\right)}$ can be at most $J$, the rank of $D_{A} F_{\left(u_{1}, w_{L \hat{\jmath}}^{1}\right)}$ can be at most $S J$, the rank of $D_{w} F_{\left(u_{1}, w_{L \hat{J}}^{1}\right)}$ can be at most $n I$, and by assumption for every $j$ we have

$\operatorname{rank} D_{\left(p_{0}, p_{1}, q\right)} F_{\left(u_{1}, w_{L J}^{1}\right)}^{j}\left(\left(\bar{p}_{0}^{1}, \bar{p}_{1}^{1}, \bar{q}^{1}\right), \ldots,\left(\bar{p}_{0}^{n I+S J+J+1}, \bar{p}_{1}^{n I+S J+J+1}, \bar{q}^{n I+S J+J+1}\right)\right)$ $\leq(L-1)(S+1)+\hat{J}-1$.

So, we can conclude that the rank of $D F_{\left(u_{1}, w_{L \hat{J}}^{1}\right)}$ can at most be $(n I+S J+J+1)((L-1)(S+1)+\hat{J}-1)+n I+J+S J=$
$(n I+S J+J+1)((L-1)(S+1)+\hat{J})-n I-S J-J-1+n I+J+S J=$ 
$(n I+S J+J+1)((L-1)(S+1)+\hat{J})-1$

But 0 being a regular value of $F_{\left(u_{1}, w_{L \hat{J}}^{1}\right)}$, requires that rank $D F_{\left(u_{1}, w_{L \hat{J}}^{1}\right)}^{j}=(n I+$ $S J+J+1)((L-1)(S+1)+\hat{J})$.

Thus, if there are at least $n I+S J+J+1$ critical equilibria, then, 0 is not a regular value of $F_{\left(u_{1}, w_{L \hat{J}}^{1}\right)}$. But since

$F_{\left(u_{1}, w_{L \hat{J}}^{1}\right)}\left(\left(\bar{p}_{0}^{1}, \bar{p}_{1}^{1}, \bar{q}^{1}\right), \ldots,\left(\bar{p}_{0}^{n I+S J+J+1}, \bar{p}_{1}^{n I+S J+J+1}, \bar{q}^{n I+S J+J+1}\right)\right)=0$

this is in contradiction with the lemma 3. 


\section{References}

1. C. Aliprantis and K. Border, "Infinite Dimensional Analysis, "SpringerVerlag, 1994.

2. Allen, On the finiteness of the Equilibrium Price Set, CARESS Working Paper No 85-03, University of Pennsylvania, Philadelphia, 1984.

3. B. Allen, Continuous Random Selections from the Utility Correspondence, CARESS Working Paper No 85-25, University of Pennsylvania, Philadelphia, 1985.

4. B. Allen, Randomization and the Limit Points of Monopolistic Competition, J. Math. Econ. 23 (1994), 205-218.

5. F. Allen and D. Gale, Optimal Security Design, Rev. Finan. Stud. 1 (1988), 229-263.

6. Y. Balasko and Cass, The Structure of Financial Equilibrium with Exogenous Yields: The case of Incomplete Markets, Econometrica 57(1) (1989), 135-163.

7. P. Billingsley, "Probability and Measure," John Wiley and Sons, 1985.

8. A. Bisin, General Equilibrium with Endogenously Incomplete Financial Markets, J. Econ. Theory 82 (1998), 19-45.

9. L.Braido, General Equilibrium with Endogenous Securities and Moral Hazard, Economic Theory.

10. G. Debreu, Existence of Competitive Equilibrium, in "Handbook of Mathematical Economics" (K. Arrow and M. Intriligator, Eds.), vol. 2, chapter 15, North Holland, Amsterdam, 1982.

11. G. Demage and G. Laroque, Private Information and the Design of Securities, J. Econ. Theory 65 (1995), 233-257.

12. D. Duffie and M. Jackson, Optimal Innovation of Futures Contracts, Rev. Finan. Stud. 2 (1989), 275-296. 
13. D. Duffie and R. Rahi, Financial Market Innovation and Security Design: An Introduction, J. Econ. Theory 65 (1995), 1-42.

14. D. Duffie and W. Shafer, Equilibrium in Incomplete Markets I: Basic Model of Generic Existence, J. Math. Econ. 14 (1985), 285-300.

15 M. Faias, "Ensaios na Teoria da Concorrência e na Teoria dos Mercados Financeiros." Ph.D. thesis, Universidade Nova de Lisboa, November 2000.

16. M. Faias, E. Moreno-Garcia and M. Páscoa, Real Indeterminacy of Equilibria and Manipulability, J. Math. Econ. 37 (2002), 325-340.

17. D. Fundenberg, and J. Tirole, "Game Theory," The MIT Press, Massachusetts Institute of Technology, 1995.

18. J. Geanakoplos, An Introduction to General Equilibrium with Incomplete Asset Markets, J. Math. Econ. 19 (1990), 1-38.

19. J. Geanakoplos and Mas-Colell, Real Indeterminacy with Financial Assets, J. Econ. Theory 47 (1989), 22-38.

20. J. Geanakoplos and H. Polemarchakis, Existence, Regularity and Constrained Sub-optimality of Competitive Allocations when Asset Market is Incomplete", in W. Heller, R. Ross and D. Starrett (eds), Uncertainty, Information, and Communication. Essays in Honor of Kenneth Arrow, Vol. 3, Cambridge University Press, Cambridge.

21. P. Halmos, " Measure theory," Springer-Verlag, Berlin, 1950.

22. C. Hara, Commission-Revenue Maximization in a General Equilibrium Model of Asset Creation, J. Econ. Theory 65 (1995),

23. O. Hart, On the Optimality of Equilibrium when Market Structure is Incomplete, J. Econ. Theory 11 (1975), 418-443.

24. M. W. Hirsch, "Differential Topology," Springer-Verlag, New York, 1976.

25. M. Magill and W. Shafer, Incomplete Markets, in W. Hildenbrand and H. Sonnesnschein (eds), Handbook of Mathematical Economics, IV, North Holland, Amsterdam, 1991. 
26. A. Mas-Colell, "The Theory of General Equilibrium: A Diferencial Approach," Cambridge University Press, Cambridge, U.K., 1985.

27. A. Mas-Colell and J. Nachbar, On the Finiteness of the Number of Critical Equilibria, with an application to random selections, J. Math. Econ. 20 (1991), 397-409.

28. P. Milgrom and R. Weber, Distributional Strategies for Games with Incomplete Information, Math Operations Res 10 (1985) 619-632.

29. W. Pesendorfer, Financial Innovation in a General Equilibrium Model, J. Econ. Theory, 65 (1995), 79-116.

30. R. Radner, Existence of Equilibrium of Plan, Prices, and Price Expectations, Econometrica 40(2) (1972), 289-303.

31. H. Royden, "Real Analysis", 2nd edn. Macmillan, New York, 1968.

32. L. Simon and W. Zame, Discontinuous Games and Endogenous Sharing Rules, Econometrica 58 (1990), 861-872.

33. H. Stahn, On Monopolistic Equilibria with Incomplete Markets: the Case of an Exchange Economy, J. Math. Econ. 29(1) (1998), 83-107.

34. J. Werner, Equilibrium in Economies with Incomplete Financial Markets, J. Econ. Theory 36 (1985), 110-119. 